\title{
Naturally Occurring Genetic Variants in Human Chromogranin A (CHGA) Associated with Hypertension as well as Hypertensive Renal Disease
}

\author{
Yuqing Chen · Fangwen Rao $\cdot$ Gen Wen · Jiaur R. Gayen $\cdot$ Kuixing Zhang $\cdot$ \\ Sucheta M. Vaingankar • Nilima Biswas $\cdot$ Manjula Mahata $\cdot$ Ryan S. Friese • \\ Maple M. Fung • Rany M. Salem · Caroline Nievergelt • Vibha Bhatnagar • \\ Vivian Y. Hook • Michael G. Ziegler • Sushil K. Mahata • Bruce A. Hamilton • \\ Daniel T. O'Connor
}

Received: 11 May 2010/ Accepted: 7 September 2010/Published online: 9 November 2010

(C) The Author(s) 2010. This article is published with open access at Springerlink.com

\begin{abstract}
Chromogranin A (CHGA) plays a fundamental role in the biogenesis of catecholamine secretory granules. Changes in storage and release of CHGA in clinical and experimental hypertension prompted us to study whether genetic variation at the $C H G A$ locus might contribute to alterations in autonomic function, and hence hypertension and its target organ consequences such as hypertensive renal disease (nephrosclerosis). Systematic polymorphism discovery across the human $C H G A$ locus revealed both common and unusual variants in both the open reading frame
\end{abstract}

A commentary to this article can be found at doi:

10.1007/s10571-010-9607-8.

\section{Y. Chen}

Renal Division, Peking University First Hospital,

Beijing 100034, China

F. Rao - G. Wen · J. R. Gayen - K. Zhang ·

S. M. Vaingankar - N. Biswas - M. Mahata .

R. S. Friese - M. M. Fung - R. M. Salem - C. Nievergelt ·

V. Bhatnagar $\cdot$ M. G. Ziegler $\cdot$ S. K. Mahata

B. A. Hamilton · D. T. O’Connor ( $\square)$

Department of Medicine (0838), UCSD School of Medicine and

VASDHS, 9500 Gilman Drive, La Jolla, CA 92093-0838, USA

e-mail: doconnor@ucsd.edu

URL: http://hypertension.ucsd.edu/

\section{Y. Hook · D. T. O'Connor}

Department of Pharmacology, University of California

at San Diego, La Jolla, CA, USA

B. A. Hamilton · D. T. O'Connor

Institute for Genomic Medicine, University of California

at San Diego, La Jolla, CA, USA

M. M. Fung · S. K. Mahata · D. T. O'Connor

VA San Diego Healthcare System, La Jolla, CA, USA and such regulatory regions as the proximal promoter and 3'-UTR. In chromaffin cell-transfected CHGA 3 '-UTR and promoter/luciferase reporter plasmids, the functional consequences of the regulatory/non-coding allelic variants were documented. Variants in both the proximal promoter and the 3'-UTR displayed statistical associations with hypertension. Genetic variation in the proximal $C H G A$ promoter predicted glomerular filtration rate in healthy twins. However, for hypertensive renal damage, both end-stage renal disease and rate of progression of earlier disease were best predicted by variants in the $3^{\prime}$-UTR. Finally, mechanistic studies were undertaken initiated by the clue that $C H G A$ promoter variation predicted circulating endothelin-1. In cultured endothelial cells, CHGA triggered co-release of not only the vasoconstrictor and pro-fibrotic endothelin-1, but also the pro-coagulant von Willebrand Factor and the pro-angiogenic angiopoietin-2. These findings, coupled with stimulation of endothelin-1 release from glomerular capillary endothelial cells by CHGA, suggest a plausible mechanism whereby genetic variation at the $C H G A$ locus eventuates in alterations in human renal function. These results document the consequences of genetic variation at the $C H G A$ locus for cardiorenal disease and suggest mechanisms whereby such variation achieves functional effects.

Keywords CHGA · Hypertension · Hypertensive nephrosclerosis

$\begin{array}{ll}\text { Abbreviations } & \\ \text { 3'-UTR } & \text { 3'-Untranslated region (of mRNA) } \\ \text { 5-HT } & \text { Serotonin (5-hydroxy-tryptamine) } \\ \text { BP } & \text { Blood pressure }\end{array}$




$\begin{array}{ll}\text { CHGA, Chga } & \begin{array}{l}\text { Chromogranin A (uppercase: human, } \\ \text { lowercase: rodent) } \\ \text { Chromogranin B (uppercase: human, }\end{array} \\ \text { DBP } & \begin{array}{l}\text { lowercase: rodent) } \\ \text { Diastolic blood pressure }\end{array} \\ \text { EDN1 } & \text { Endothelin-1 } \\ \text { ESRD } & \text { End stage renal disease } \\ \text { GFR } & \text { Glomerular filtration rate } \\ \text { siRNA } & \text { Small interfering RNA } \\ \text { SBP } & \text { Systolic blood pressure } \\ \text { TH } & \text { Tyrosine hydroxylase } \\ \text { vWF } & \text { von Willebrand factor }\end{array}$

\section{Introduction}

Chromogranin A (CHGA: human, Chga: rodent) is the major protein co-stored and co-released with catecholamines from secretory vesicles in adrenal medulla and postganglionic sympathetic axons (Takiyyuddin et al. 1990b). CHGA is required for formation of catecholamine secretory vesicles in chromaffin cells (Mahapatra et al. 2005) and its expression may be sufficient even to induce a regulated secretory system in non-secretory cells (Kim et al. 2001). CHGA is also a pro-hormone that gives rise to biologically active peptides such as the dysglycemic peptide pancreastatin (Cadman et al. 2002; Tatemoto et al. 1986), the antimicrobial peptide chromacin (Strub et al. 1996), the vasodilator vasostatin, and catestatin that acts to inhibit catecholamine release (Mahata et al. 1999, 1997). Proteolytic enzymes specifically catalyzing CHGA cleavage to active peptides have been identified including the pro-hormone convertases (Eskeland et al. 1996), plasmin (Jiang et al. 2001; Parmer et al. 2000; Biswas et al. 2008), and cathepsin L (Biswas et al. 2009).

Over the past $\sim 20$ years, phenotypic links between CHGA and hereditary (essential, idiopathic, and genetic) human (Hsiao et al. 1991; O'Connor 1985; Takiyyuddin et al. 1990a) and rodent (O'Connor et al. 1999; Fries et al. 2004) hypertension have been repeatedly observed. Plasma CHGA concentration correlates with catecholamine release rates (Dimsdale et al. 1992), and increases in blood pressure caused by the action of catecholamines may be coupled to the formation of dense-core secretory granules, whose biogenesis is regulated in vivo by CHGA (Mahapatra et al. 2005). Increased serum CHGA has been detected not only in patients with essential hypertension (O'Connor 1985) but also hypertensive consequences such as cardiac or renal (Hsiao et al. 1990) failure. Intriguingly, targeted ablation of the Chga locus in the mouse also results in elevated BP (Mahapatra et al. 2005) and global autonomic dysfunction, both sympathetic and parasympathetic (Gayen et al. 2009); recently, using both knockout and replacement strategies, we established that Chga gene copy number exhibits a biphasic (or "U-shaped") effect on both BP and catecholamine release (Vaingankar et al. 2010a, b); we observed parallel findings on CHGA secretion and autonomic stress responses in humans (Vaingankar et al. 2010a, b), suggesting that an optimal amount of CHGA may be required to establish appropriate catecholamine storage and release, and hence BP homeostasis.

We systematically identified genetic variation across the human $C H G A$ locus by resequencing the gene in several human populations (Wen et al. 2004). We then explored whether common genetic variation at the CHGA locus is associated with hypertension or hypertensive complications such as nephrosclerosis beginning with large populationbased samples. We then established an influence on an earlier pathogenic phenotype (environmental stress-evoked change in BP), and finally documented the effects on gene expression in transfected reporter systems in chromaffin cells.

\section{Chromogranin A Genetic Variants and Hypertension}

A common haplotype of the $C H G A$ proximal promoter region, CGATA (at T-1014C, T-988G, G-462A, C-415T, and A-89C), blunted the BP response to cold stress, and the response exhibited molecular heterosis (most extreme phenotype in heterozygotes) between the two most common promoter haplotypes (CGATA/TTGTC) (Chen et al. 2008a). Homozygosity for the minor alleles at T-1014C $(\mathrm{C} / \mathrm{C}), \mathrm{T}-988 \mathrm{G}(\mathrm{G} / \mathrm{G})$, and G-462A (A/A) predicted lower stress BP increments with peak prediction for G-462A (rs9658634) heterozygotes ( 6-7 mmHg). G-462A also predicted resting $\mathrm{BP}$ in the population, accounting for $\sim 3 / 2 \mathrm{mmHg}$ SBP/DBP, once again with the appearance of molecular heterosis (i.e., highest BP in G/A heterozygotes). In transfected CHGA promoter/luciferase reporters, CGATA had diminished expression compared to TTGTC, under both basal conditions and after secretory activation by pre-ganglionic stimuli (nicotinic cholinergic, VIP). Variant G-462A altered a COUP-TF transcriptional control motif and the two alleles in transfected promoters differed in basal activity $(\mathrm{G}>\mathrm{A})$, as well as the response to COUP-II-TF $(A>G)$ and retinoic acid $(G>A)$. Findings of molecular heterosis were also demonstrated for the transfected $C H G A$ promoter in cella, wherein the diploid combination of the two alleles at G-462A (G/A heterozygosity) gave rise to greater luciferase expression than either allele in isolation (Chen et al. 2008a).

A common ( $\sim 27 \%$ allele frequency) genetic variant in the CHGA 3'-UTR (C + 87T; rs7610) was strongly associated with human essential hypertension, accounting for up 
to $\sim 12 / 9 \mathrm{mmHg}$ of BP in men, or $\sim 1.9 / 1.2 \%$ of population SBP/DBP variance, especially in males (Chen et al. 2008b). The 3'-UTR variant also predicts environmental stressinduced increments in blood pressure: the same allele $(+87 \mathrm{~T})$ that diminished basal BP in the population also decreased the SBP response to stress by $\sim 12 \mathrm{mmHg}$ $(P=0.017)$, and the response was smaller in women (by $\sim 6 \mathrm{mmHg}, P=0.009$ ), suggesting a mechanism for early effects of the gene on a pathogenic series of events eventuating in sustained blood pressure elevation. The $3^{\prime}$-UTR variant is in a region of sequence conservation across species, and acts to change $C H G A$ gene expression in chromaffin cells. In a chromaffin cell-transfected $C H G A 3^{\prime}$-UTR/ luciferase reporter plasmid, the $+87 \mathrm{~T}$ allele associated with lower BP also decreased reporter expression by $\sim 30 \%$ $(P=0.009)$. In cultured chromaffin cells, reducing endogenous Chga expression by siRNA caused $\sim 2 / 3$ depletion of catecholamine storage vesicles $(P<0.0001)$. At multiple levels (CHGA expression, heritable circulatory response to environmental stress, and finally basal BP in the population), sex seemed to play an important role in mediating the effect of genetic variation on phenotype (Chen et al. 2008b).

\section{Chromogranin A Genetic Variants: Renal Function and Hypertensive Nephrosclerosis}

In African-Americans with a clinical diagnosis of hypertensive renal disease (or nephrosclerosis) (Salem et al. 2008), genetic variation at $C H G A$ predicts risk for developing the trait, and the peak effect lies in haplotypes spanning the $3^{\prime}$-end of the gene.

In a large twin pair sample with normal renal function, glomerular filtration rate (GFR) was highly heritable (at $h^{2}=78 \pm 3 \%, P<10^{-25}$ ), and $C H G A$ common promoter haplotypes (G-1106A, rs9658628; A-1018T, rs9658629; T-1014C, rs9658630; T-988G, rs9658631; G-462A, rs9658634; T-415C, rs9658635; C-89A, rs7159323; C-57T, rs9658638) predicted GFR: haplotype-2 (GATT GTCC) copy number was associated with higher GFR, while haplotype-3 (GACGATAC) predicted lower GFR (Chen et al. 2009).

In subjects from the NIDDK AASK (African-American Study of Kidney Disease and Hypertension) trial, the chronic decline rate of GFR was influenced by genetic variation at CHGA, with the most prominent effect $(P=0.006)$ from haplotype-1 in the $3^{\prime}$-haplotype block, spanning $3^{\prime}$-UTR $\mathrm{C}+87 \mathrm{~T} / \mathrm{rs} 7610$ (Chen et al. 2009). The effect was also seen for diploid haplotype pairs in that block ( $P=0.007)$.

We first noted association of $C H G A$ genetic variation with circulating endothelin-1 (EDN1) (Lillie et al. 2007), and then found that $C H G A$ genetic variation or secretion predicted not only EDN1 but also renal traits in a large series of twin and sibling pairs (Chen et al. 2009). Plasma CHGA positively correlated with EDN1 and negatively correlated with GFR. Thus we explored the possible mechanisms of CHGA effects on GFR as well as progression of kidney disease in culture cells. CHGA released EDN1 from HUVEC (human umbilical vein endothelial cells), dependent upon extracellular $\mathrm{Ca}^{2+}$ influx through voltage-operated $\mathrm{Ca}^{2+}$ channels, and inhibited by the 5-HT (serotonin) antagonist cyproheptadine. The response was also triggered by the CHGA synthetic amino terminus (CHGA1-40). CHGA triggered endothelial co-release of not only EDN1, but also von Willebrand Factor (vWF), and angiopoietin-2, consistent with a global Weibel Palade Body (i.e., endothelial secretory granule) exocytosis response to CHGA. In renal glomerular cells, CHGA caused secretion of EDN1 from glomerular endothelial cells, and induced secretion of TGF-beta-1 from mesangial cells co-cultured with glomerular endothelial cells, along with TGF-beta-1 signal transduction (Chen et al. 2009).

In this series of studies of CHGA genetic variation on disease, especially hypertension and hypertensive nephrosclerosis, we took advantage of multiple resources and approaches, including intermediate phenotypes (biochemical and/or physiological traits proceeding disease phenotypes), different populations with replication, as well as functional experiments in cella, to identify the pathways whereby CHGA influences disease risk. Many of these observations are consistent with the "common disease/ common allele" hypothesis for frequent traits in the population, and suggest new molecular strategies for probing the pathophysiology, risk, and rational treatment of hypertension and hypertensive nephrosclerosis. Other observations, such as those on the unusual (minor allele frequency $\sim 6 \%$ ) CHGA coding variant Gly364Ser, in the catecholamine release-inhibitory "catestatin" region of the protein, indicate a role for rare variants in prediction of cardiovascular risk (Rao et al. 2007).

\section{"Intermediate" Phenotypes}

In the setting of late penetrance of the ultimate disease trait (such as hypertension), as well as likely genetic heterogeneity, the "intermediate" phenotype (Lillie and O'Connor 2006; Shih and O'Connor 2008) strategy may be a useful approach in the search for disease predisposition loci. Autonomic traits with heritable determination may be of particular value in investigation of the genetic underpinnings of hypertension. In accordance with this pathway concept, we pursued intermediate traits in these studies. Secretion of CHGA, estimated by its plasma concentration, as well as the hemodynamic response to environmental 
stressors (such as cold), may be predictors of the development of later cardiovascular events, such as hypertension (Markovitz et al. 1998; Menkes et al. 1989; Schneider et al. 2003; Snieder et al. 2002; Treiber et al. 2003). Such responses, occurring even prior to the onset of disease, would be useful biochemical or physiological "intermediate" phenotypes in probing the genetic determinants of hypertension (Lillie and O'Connor 2006; O'Connor et al. 2000, 2002). When we analyzed the cold stress response in our twin subjects, we found that that both change in BP and final (post-stress) BP are heritable, and thus may constitute valuable intermediate phenotypic anchor points for hypertension (Chen et al. 2008a, b).

\section{Molecular Heterosis}

The CHGA common promoter haplotype homozygosity (CGATA/CGATA) and individual SNP G-462A effects were more extreme for heterozygotes, suggesting "molecular heterosis". The phenomenon of molecular heterosis has been defined as occurring when polymorphism at a single genetic locus displays a significantly greater or lesser effect on a quantitative trait than homozygosity at the same locus. While the phenomenon may initially seem counter-intuitive, it may be explained by one of several underlying mechanisms, including a U-shaped dose-response relationship for gene-on-trait, greater "fitness" in heterozygotes, or hidden stratification in one homozygote class. One such stratification might be the effect of allelic variation at other (nonCHGA) trans-QTLs on cold stress and basal BPs, such as the associations we have reported for polymorphisms at tyrosine hydroxylase (TH) (Zhang et al. 2010a, b; Rao et al. 2007) or Rho kinase (ROCK2) (Seasholtz et al. 2006).

\section{Sex: Role in Hypertension and Intermediate Phenotypes}

In the wake of the sex-dependent effect of $C H G A$ genetic variation on $\mathrm{BP}$, we question potential interactions of gene and sex, and for CHGA we found that sex played a role at each of several steps: biochemical, physiological, and disease levels. Why adrenergic genetic variations yield such different consequences in men and women? Acute vascular responses to adrenergic stimuli are sex-dependent, and the long-term consequences of repeated stressors on resting blood pressure or the late appearance hypertension differ by sex (Markovitz et al. 1998).

More recently, we established that common promoter variation at two adrenergic loci, $C H G B$ and $T H$ (Zhang et al. 2010a, b), disrupted DNA sequence matches for the transcription factor SRY (Sex-determining Region Y), a Y-chromosome-encoded HMG (high mobility group)-box factor that may be instrumental in generating the male phenotype, but which also has effects on gene expression in the adult organism.

\section{Chromogranin A and the Kidney: Potential Clinical Implications}

Since CHGA is important in storage and release of catecholamines (Mahapatra et al. 2005; Vaingankar et al. 2010a, b), and is associated with hypertension (Chen et al. 2009, 2008a), it was reasonable to explore whether CHGA might have an effect on hypertensive nephrosclerosis, via catecholamines or hypertension. In our initial study of predominantly healthy twin pairs (Lillie et al. 2007), we found that $C H G A$ common genetic variation, especially in the promoter region, predicted circulating EDN1 concentration.

We then found that $C H G A$ polymorphism predicted the occurrence of hypertensive renal disease in AfricanAmericans with the peak risk conferred by variation in the 3 '-region of the gene (Salem et al. 2008).

We finally proceeded to more mechanistic studies (Chen et al. 2009), attempting to understand the links between CHGA genetic variation, EDN1, and renal function. Here CHGA displayed effects on GFR in healthy individuals, as well as GFR decline rate in subjects with progressive of renal disease, and the effects seemed independent of blood pressure. EDN1 suggested a candidate pathway for exploration: we then identified CHGA-induced release of other endothelial mediators that could influence renal function, including the vasoconstrictor and pro-fibrotic EDN1, but also the pro-coagulant $\mathrm{vWF}$ and the pro-angiogenic angiopoietin-2. Co-release of all the three by CHGA from endothelial cells was consistent with a mechanism whereby CHGA triggered exocytosis of the endothelial cell secretory granule: the Weibel Palade Body.

Why did a promoter block of $C H G A$ best predict GFR in healthy individuals, while a $3^{\prime}$ block best predicted GFR decline in subjects with renal dysfunction (Chen et al. 2009)? Transfected luciferase reporter studies of genetic variants in these $C H G A$ domains have now established that variants in both the proximal promoter (Chen et al. 2008a) and the 3'-UTR (Chen et al. 2008b) are functional, and thus capable of influencing disease traits. In our previous case/ control studies of hypertensive renal disease, 3'-UTR variation was associated with ESRD (Salem et al. 2008), while promoter variation resulting in alterations of nuclear hormone receptor trans-activation may influence blood pressure (Chen et al. 2008a).

\section{Conclusion and Perspectives}

Thus, common genetic variation across the human $C H G A$ locus is associated with cardio-renal disease traits as well 
as their precursor (or "intermediate") phenotypes. The associated variants are functional when tested in cellular systems. Such findings may lead to novel approaches to the pathophysiology, diagnosis, and treatment of the autonomic dysfunction predisposing to such disease traits.

Open Access This article is distributed under the terms of the Creative Commons Attribution Noncommercial License which permits any noncommercial use, distribution, and reproduction in any medium, provided the original author(s) and source are credited.

\section{References}

Biswas N, Vaingankar SM, Mahata M, Das M, Gayen JR, Taupenot L et al (2008) Proteolytic cleavage of human chromogranin a containing naturally occurring catestatin variants: differential processing at catestatin region by plasmin. Endocrinology 149: $749-757$

Biswas N, Rodriguez-Flores JL, Courel M, Gayen JR, Vaingankar SM, Mahata M et al (2009) Cathepsin L colocalizes with chromogranin a in chromaffin vesicles to generate active peptides. Endocrinology 150:3547-3557

Cadman PE, Rao F, Mahata SK, O'Connor DT (2002) Studies of the dysglycemic peptide, pancreastatin, using a human forearm model. Ann NY Acad Sci 971:528-529

Chen Y, Rao F, Rodriguez-Flores JL, Mahapatra NR, Mahata M, Wen $G$ et al (2008a) Common genetic variants in the chromogranin A promoter alter autonomic activity and blood pressure. Kidney Int 74:115-125

Chen Y, Rao F, Rodriguez-Flores JL, Mahata M, Fung MM, Stridsberg M et al (2008b) Naturally occurring human genetic variation in the $3^{\prime}$-untranslated region of the secretory protein chromogranin $\mathrm{A}$ is associated with autonomic blood pressure regulation and hypertension in a sex-dependent fashion. J Am Coll Cardiol 52:1468-1481

Chen Y, Mahata M, Rao F, Khandrika S, Courel M, Fung MM et al (2009) Chromogranin A regulates renal function by triggering Weibel-Palade body exocytosis. J Am Soc Nephrol 20: $1623-1632$

Dimsdale JE, O'Connor DT, Ziegler M, Mills P (1992) Chromogranin A correlates with norepinephrine release rate. Life Sci 51:519-525

Eskeland NL, Zhou A, Dinh TQ, Wu H, Parmer RJ, Mains RE, O'Connor DT (1996) Chromogranin A processing and secretion: specific role of endogenous and exogenous prohormone convertases in the regulated secretory pathway. J Clin Invest 98: $148-156$

Fries RS, Mahboubi P, Mahapatra NR, Mahata SK, Schork NJ, Schmid-Schoenbein GW, O'Connor DT (2004) Neuroendocrine transcriptome in genetic hypertension: multiple changes in diverse adrenal physiological systems. Hypertension 43: $1301-1311$

Gayen JR, Gu Y, O'Connor DT, Mahata SK (2009) Global disturbances in autonomic function yield cardiovascular instability and hypertension in the chromogranin a null mouse. Endocrinology 150:5027-5035

Hsiao RJ, Mezger MS, O'Connor DT (1990) Chromogranin A in uremia: progressive retention of immunoreactive fragments. Kidney Int 37:955-964

Hsiao RJ, Parmer RJ, Takiyyuddin MA, O’Connor DT (1991) Chromogranin A storage and secretion: sensitivity and specificity for the diagnosis of pheochromocytoma. Medicine (Baltimore) 70:33-45
Jiang Q, Taupenot L, Mahata SK, Mahata M, O'Connor DT, Miles LA, Parmer RJ (2001) Proteolytic cleavage of chromogranin A $(\mathrm{CgA})$ by plasmin. Selective liberation of a specific bioactive $\mathrm{CgA}$ fragment that regulates catecholamine release. J Biol Chem 276:25022-25029

Kim T, Tao-Cheng JH, Eiden LE, Loh YP (2001) Chromogranin A, an "on/off" switch controlling dense-core secretory granule biogenesis. Cell 106:499-509

Lillie EO, O'Connor DT (2006) Early phenotypic changes in hypertension: a role for the autonomic nervous system and heredity. Hypertension 47:331-333

Lillie EO, Mahata M, Khandrika S, Rao F, Bundey RA, Wen G et al (2007) Heredity of endothelin secretion: human twin studies reveal the influence of polymorphism at the chromogranin A locus, a novel determinant of endothelial function. Circulation 115:2282-2291

Mahapatra NR, O'Connor DT, Vaingankar SM, Hikim AP, Mahata M, Ray S et al (2005) Hypertension from targeted ablation of chromogranin A can be rescued by the human ortholog. J Clin Invest 115:1942-1952

Mahata SK, O'Connor DT, Mahata M, Yoo SH, Taupenot L, Wu H et al (1997) Novel autocrine feedback control of catecholamine release. A discrete chromogranin a fragment is a noncompetitive nicotinic cholinergic antagonist. J Clin Invest 100:1623-1633

Mahata SK, Mahata M, Parmer RJ, O'Connor DT (1999) Desensitization of catecholamine release. The novel catecholamine release-inhibitory peptide catestatin (chromogranin A344-364) acts at the receptor to prevent nicotinic cholinergic tolerance. J Biol Chem 274:2920-2928

Markovitz JH, Raczynski JM, Wallace D, Chettur V, Chesney MA (1998) Cardiovascular reactivity to video game predicts subsequent blood pressure increases in young men: The CARDIA study. Psychosom Med 60:186-191

Menkes MS, Matthews KA, Krantz DS, Lundberg U, Mead LA, Qaqish B et al (1989) Cardiovascular reactivity to the cold pressor test as a predictor of hypertension. Hypertension 14: $524-530$

O'Connor DT (1985) Plasma chromogranin A. Initial studies in human hypertension. Hypertension 7:176-179

O’Connor DT, Takiyyuddin MA, Printz MP, Dinh TQ, Barbosa JA, Rozansky DJ et al (1999) Catecholamine storage vesicle protein expression in genetic hypertension. Blood Press 8:285-295

O'Connor DT, Insel PA, Ziegler MG, Hook VY, Smith DW, Hamilton BA et al (2000) Heredity and the autonomic nervous system in human hypertension. Curr Hypertens Rep 2:16-22

O'Connor DT, Kailasam MT, Kennedy BP, Ziegler MG, Yanaihara N, Parmer RJ (2002) Early decline in the catecholamine releaseinhibitory peptide catestatin in humans at genetic risk of hypertension. J Hypertens 20:1335-1345

Parmer RJ, Mahata M, Gong Y, Mahata SK, Jiang Q, O'Connor DT et al (2000) Processing of chromogranin A by plasmin provides a novel mechanism for regulating catecholamine secretion. J Clin Invest 106:907-915

Rao F, Zhang L, Wessel J, Zhang K, Wen G, Kennedy BP et al (2007) Tyrosine hydroxylase, the rate-limiting enzyme in catecholamine biosynthesis: discovery of common human genetic variants governing transcription, autonomic activity, and blood pressure in vivo. Circulation 116:993-1006

Salem RM, Cadman PE, Chen Y, Rao F, Wen G, Hamilton BA et al (2008) Chromogranin A polymorphisms are associated with hypertensive renal disease. J Am Soc Nephrol 19:600-614

Schneider GM, Jacobs DW, Gevirtz RN, O'Connor DT (2003) Cardiovascular haemodynamic response to repeated mental stress in normotensive subjects at genetic risk of hypertension: evidence of enhanced reactivity, blunted adaptation, and delayed recovery. J Hum Hypertens 17:829-840 
Seasholtz TM, Wessel J, Rao F, Rana BK, Khandrika S, Kennedy BP et al (2006) Rho kinase polymorphism influences blood pressure and systemic vascular resistance in human twins: role of heredity. Hypertension 47:937-947

Shih PA, O'Connor DT (2008) Hereditary determinants of human hypertension: strategies in the setting of genetic complexity. Hypertension 51:1456-1464

Snieder H, Harshfield GA, Barbeau P, Pollock DM, Pollock JS, Treiber FA (2002) Dissecting the genetic architecture of the cardiovascular and renal stress response. Biol Psychol 61:73-95

Strub JM, Goumon Y, Lugardon K, Capon C, Lopez M, Moniatte M et al (1996) Antibacterial activity of glycosylated and phosphorylated chromogranin A-derived peptide 173-194 from bovine adrenal medullary chromaffin granules. J Biol Chem 271: 28533-28540

Takiyyuddin MA, Cervenka JH, Hsiao RJ, Barbosa JA, Parmer RJ, O'Connor DT (1990a) Chromogranin A. Storage and release in hypertension. Hypertension 15:237-246

Takiyyuddin MA, Cervenka JH, Sullivan PA, Pandian MR, Parmer RJ, Barbosa JA, O'Connor DT (1990b) Is physiologic sympathoadrenal catecholamine release exocytotic in humans? Circulation 81:185-195

Tatemoto K, Efendic S, Mutt V, Makk G, Feistner GJ, Barchas JD (1986) Pancreastatin, a novel pancreatic peptide that inhibits insulin secretion. Nature 324:476-478
Treiber FA, Kamarck T, Schneiderman N, Sheffield D, Kapuku G, Taylor T (2003) Cardiovascular reactivity and development of preclinical and clinical disease states. Psychosom Med 65:46-62

Vaingankar SM, Li Y, Biswas N, Gayen J, Choksi S, Rao F et al (2010a) Effects of chromogranin A deficiency and excess in vivo: biphasic blood pressure and catecholamine responses. J Hypertens 28:817-825

Vaingankar SM, Li Y, Corti A, Biswas N, Gayen J, O'Connor DT, Mahata SK (2010b) Long human CHGA flanking chromosome 14 sequence required for optimal BAC transgenic "rescue" of disease phenotypes in the mouse Chga knockout. Physiol Genomics 41:91-101

Wen G, Mahata SK, Cadman P, Mahata M, Ghosh S, Mahapatra NR et al (2004) Both rare and common polymorphisms contribute functional variation at CHGA, a regulator of catecholamine physiology. Am J Hum Genet 74:197-207

Zhang K, Rao F, Wang L, Rana BK, Ghosh S, Mahata M et al (2010a) Common functional genetic variants in catecholamine storage vesicle protein promoter motifs interact to trigger systemic hypertension. J Am Coll Cardiol 55:1463-1475

Zhang K, Zhang L, Rao F, Brar B, Rodriguez-Flores JL, Taupenot L, O'Connor DT (2010b) Human tyrosine hydroxylase natural genetic variation: delineation of functional transcriptional control motifs disrupted in the proximal promoter. Circ Cardiovasc Genet 3:187-198 